1 Hacettepe Journal of Mathematics and Statistics

h Volume 46 (3) (2017), 419-426

\title{
Fixed point theory on spaces with vector-valued metrics and application
}

\author{
Bazine Safia* ${ }^{*}$ Ellaggoune Fateh ${ }^{\dagger}$ and Aliouche Abdelkrim ${ }^{\ddagger}$
}

\begin{abstract}
The purpose of this work is to prove some common fixed point theorems for two operators on a set endowed with one or two vector-valued metrics. The use of vector-valued metrics makes it possible for each equation of a system to have its own Lipschitz property, while the use of two such metrics makes it possible for the Lipschitz condition to be expressed with respect to an incomplete metric. An application is presented for a system of operatorial equations.
\end{abstract}

Keywords: Fixed point, vector-valued metric, matrix convergent to zero.

2000 AMS Classification: $37 \mathrm{H} 10,54 \mathrm{H} 25$.

Received : 20.01.2016 Accepted : 21.06.2016 Doi : 10.15672/HJMS.20164519331

\section{Introduction}

Fixed point theory plays a basic role in applications of many branches of mathematics. Finding a fixed point of contractive mappings becomes the center of strong research activity. The classical Banach contraction principle was extended for contraction mappings on spaces endowed with vector-valued metrics by Perov in 1964 (see [4]). The starting point of this work was the article of D. O'Regan, R. Precup (see [3]). In the first section we present some results for two operators on a generalized metric space and in the second section we will use two generalized metrics.

\footnotetext{
*Department of mathematics, University of Larbi Ben M'hidi, Oum El Bouaghi, Algeria, Email: bazine-safia@hotmail.fr

$\dagger$ Department of mathematics, University of 8 mai 1945, Guelma, Algeria, Email: fellaggoune@gmail.com

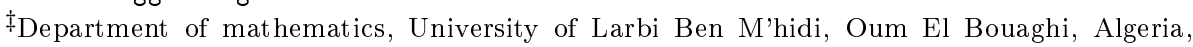
Email: alioumathøyahoo.fr
} 


\section{Basic definitions and auxiliary results}

The aim of this section is to present some definitions and results that will be needed in the sequel.

2.1. Definition. Let $X$ be a nonempty set. A mapping $d: X \times X \rightarrow \mathbb{R}_{+}^{n}$ is called a vector-valued metric on $X$ if the following properties are satisfied:

(1) $d(x, y)=0$ if and only if $x=y$ for all $x, y \in X$;

(2) $d(x, y)=d(y, x)$ for all $x, y \in X$;

(3) $d(x, z) \leq d(x, y)+d(y, z)$ for all $x, y, z \in X$.

If $\alpha, \beta \in \mathbb{R}^{n}$ with $\alpha=\left(\alpha_{1}, \alpha_{2}, \ldots, \alpha_{n}\right), \beta=\left(\beta_{1}, \beta_{2}, \ldots, \beta_{n}\right)$ and $c \in \mathbb{R}$, then by $\alpha \leq \beta$ (respectively $\alpha<\beta$ ), we mean that $\alpha_{i} \leq \beta_{i}$ (respectively $\alpha_{i}<\beta_{i}$ ), for $i \in\{1, \ldots, n\}$ and by $\alpha \leq c$ we mean that $\alpha_{i} \leq c$, for $i \in\{1, \ldots, n\}$.

A set $X$ equipped with a vector-valued metric $d$ is called a generalized metric space. We will denote such a space with $(X, d)$. For the generalized metric spaces, the notions of convergent sequence, Cauchy sequence, completeness, open subset and closed subset are similar to those for usual metric spaces.

2.2. Definition. If $f: X \rightarrow X$ is a single-valued operator, then we denote by Fix $(f)$ the set of all fixed points of $f$; that is, Fix $(f)=\{x \in X \mid x=f(x)\}$.

2.3. Definition. Let $f$ and $g$ be self-mapping of a generalized metric space $(X, d)$. An element $x \in X$ is said to be a common fixed point of $f$ and $g$ if and only if $x=f(x)=g(x)$.

Throughout this paper we denote by $\mathcal{M}_{n \times n}\left(\mathbb{R}_{+}\right)$the set of all $n \times n$ matrices with positive elements, by $\ominus$ the zero $n \times n$ matrix and by $I$ the identity $n \times n$ matrix.

2.4. Definition. [9] $A \in \mathcal{M}_{n \times n}\left(\mathbb{R}_{+}\right)$is said to be matrix convergent to zero if and only if $A^{k} \rightarrow \ominus$, as $k \rightarrow \infty$.

Notice that, for the proof of the main results, we need the following theorem, part of which being a classical result in matrix analysis; see, for example, [7] (page 37) and [9] (page 12). For the assertion (iii) see [5].

2.5. Theorem. Let $A \in \mathcal{M}_{n \times n}\left(\mathbb{R}_{+}\right)$. The following statements are equivalent:

(i): $A$ is convergent towards zero.

(ii): The eigenvalues of $A$ are in the open unit disc, that is, $|\lambda|<1$, for every $\lambda \in \mathbb{C}$ with $\operatorname{det}(A-\lambda I)=0$.

(iii): The matrix $(I-A)$ is nonsingular and

$$
(I-A)^{-1}=I+A+A^{2}+\cdots+A^{n}+\cdots .
$$

(iv): The matrix $(I-A)$ is nonsingular and $(I-A)^{-1}$ has nonnegative elements.

(v): The matrices $A q$ and $q^{T} A$ converge to zero for each $q \in \mathbb{R}^{n}$.

2.6. Example. Some examples of matrix convergent to zero are:

(a): any matrix $A=\left(\begin{array}{ll}a & a \\ b & b\end{array}\right)$, where $a, b \in \mathbb{R}_{+}$and $a+b<1$;

(b): any matrix $A=\left(\begin{array}{ll}a & b \\ a & b\end{array}\right)$, where $a, b \in \mathbb{R}_{+}$and $a+b<1$;

(c): any matrix $A=\left(\begin{array}{ll}a & b \\ 0 & c\end{array}\right)$, where $a, b, c \in \mathbb{R}_{+}$and $\max \{a, c\}<1$.

For other examples and considerations on matrices which converge to zero, see Turinici [8], Kannan [2] and Reich [6]. 
Main result for self contractions on generalized metric spaces is Perov's fixed point theorem, see [4].

2.7. Theorem. (Perov's theorem) Let $(X, d)$ be a complete generalized metric space and the mapping $f: X \rightarrow X$ with the property that there exists a matrix $A \in \mathcal{M}_{n \times n}\left(\mathbb{R}_{+}\right)$ such that

$$
d(f(x), f(y)) \leq A d(x, y)
$$

for all $x, y \in X$. If $A$ is a matrix convergent towards zero, then

(1) the mapping $f$ has a unique fixed point $z$, i.e. Fix $(f)=\{z\}$;

(2) the sequence of successive approximations $\left(x_{m}\right)_{m \in \mathbb{N}}, x_{m}=f^{m}\left(x_{0}\right)$ is convergent and it has the limit $z$, for all $x_{0} \in X$;

(3) one has the following estimation:

$$
d\left(x_{m}, z\right) \leq A^{m}(I-A)^{-1} d\left(x_{0}, x_{1}\right), \quad \text { for all } m \in \mathbb{N} ;
$$

(4) if $g: X \rightarrow X$ satisfies the condition $d(f(x), g(x)) \leq \eta$, for all $x \in X, \eta \in \mathbb{R}^{n}$ and considering the sequence $y_{m}=g^{m}\left(x_{0}\right)$ one has

$$
d\left(y_{m}, z\right) \leq(I-A)^{-1} \eta+A^{m}(I-A)^{-1} d\left(x_{0}, x_{1}\right), \quad \text { for all } m \in \mathbb{N} .
$$

Hence, it is of great interest to give fixed point results for single-valued operators on a set endowed with vector-valued metrics or norms. The use of vector-valued metrics makes it possible for each equation of a system to have its own Lipschitz property, while the use of two such metrics makes it possible for the Lipschitz condition to be expressed with respect to an incomplete metric. However, some advantages of a vector-valued norm with respect to the usual scalar norms were already pointed out by Precup in [5]. Therefore, we may conclude that for different types of estimations, the use of the vector-valued norm and, correspondingly, of the matrices convergent to zero, is more appropriate when treating systems of equations. In this paper we prove some theorems for generalized single-valued contractions on spaces endowed with vector-valued metrics. Our results are extensions of the theorems given by Perov in [4], Hardy and Rogers in [1], O'Regan and Precup in [3].

\section{Main results}

The first main result of this paper is the following:

3.1. Theorem. Let $(X, d)$ be a complete generalized metric space. Assume that the operators $f, g: X \rightarrow X$ satisfy the following conditions :

there exists matrices $M, N, P \in \mathcal{M}_{n \times n}\left(\mathbb{R}_{+}\right)$with :

(i): $(I-N-P)$ is nonsingular and $(I-N-P)^{-1} \in \mathcal{M}_{n \times n}\left(\mathbb{R}_{+}\right)$;

(ii): $C$ is convergent toward zero, where $C=(I-N-P)^{-1}(M+N+P)$;

(iii): $d(f(x), g(y)) \leq M d(x, y)+N[d(x, f(x))+d(y, g(y))]+P[d(x, g(y))+d(y, f(x))]$, for all $x, y \in X$.

Then:

(1) $f$ and $g$ have a common fixed point $z \in X$

(2) If, in addition, $(I-M-2 P)$ is nonsingular and $(I-M-2 P)^{-1} \in \mathcal{M}_{n \times n}\left(\mathbb{R}_{+}\right)$, then $z$ is a unique common fixed point of $f$ and $g$.

Proof. Let $x_{0}$ be some point in $X$, we consider $\left(x_{n}\right)_{n \in \mathbb{N}}$ the sequence of successive approximations for $f$ and $g$, defined by:

$$
\begin{array}{ll}
x_{2 n+1}=f\left(x_{2 n}\right), & n=0,1, \ldots \\
x_{2 n+2}=g\left(x_{2 n+1}\right), & n=0,1, \ldots
\end{array}
$$


We have:

$$
\begin{aligned}
d\left(x_{2 n}, x_{2 n+1}\right)= & d\left(g\left(x_{2 n-1}\right), f\left(x_{2 n}\right)\right) \\
\leq & M d\left(x_{2 n-1}, x_{2 n}\right)+N\left[d\left(x_{2 n}, f\left(x_{2 n}\right)\right)+d\left(x_{2 n-1}, g\left(x_{2 n-1}\right)\right)\right] \\
& +P\left[d\left(x_{2 n}, g\left(x_{2 n-1}\right)\right)+d\left(x_{2 n-1}, f\left(x_{2 n}\right)\right)\right] \\
= & M d\left(x_{2 n-1}, x_{2 n}\right)+N\left[d\left(x_{2 n}, x_{2 n+1}\right)+d\left(x_{2 n-1}, x_{2 n}\right)\right]+P d\left(x_{2 n-1}, x_{2 n+1}\right) \\
\leq & M d\left(x_{2 n-1}, x_{2 n}\right)+N\left[d\left(x_{2 n}, x_{2 n+1}\right)+d\left(x_{2 n-1}, x_{2 n}\right)\right] \\
& +P\left[d\left(x_{2 n-1}, x_{2 n}\right)+d\left(x_{2 n}, x_{2 n+1}\right)\right] .
\end{aligned}
$$

This implies that:

$$
d\left(x_{2 n}, x_{2 n+1}\right) \leq(I-N-P)^{-1}(M+N+P) d\left(x_{2 n-1}, x_{2 n}\right)=C d\left(x_{2 n-1}, x_{2 n}\right) .
$$

Similarly, we have:

$$
\begin{aligned}
d\left(x_{2 n+1}, x_{2 n+2}\right)= & d\left(f\left(x_{2 n}\right), g\left(x_{2 n+1}\right)\right) \\
\leq & M d\left(x_{2 n}, x_{2 n+1}\right)+N\left[d\left(x_{2 n}, f\left(x_{2 n}\right)\right)+d\left(x_{2 n+1}, g\left(x_{2 n+1}\right)\right)\right] \\
& +P\left[d\left(x_{2 n}, g\left(x_{2 n+1}\right)\right)+d\left(x_{2 n+1}, f\left(x_{2 n}\right)\right)\right] \\
= & M d\left(x_{2 n}, x_{2 n+1}\right)+N\left[d\left(x_{2 n}, x_{2 n+1}\right)+d\left(x_{2 n+1}, x_{2 n+2}\right)\right]+P d\left(x_{2 n}, x_{2 n+2}\right) \\
\leq & M d\left(x_{2 n}, x_{2 n+1}\right)+N\left[d\left(x_{2 n}, x_{2 n+1}\right)+d\left(x_{2 n+1}, x_{2 n+2}\right)\right] \\
& +P\left[d\left(x_{2 n}, x_{2 n+1}\right)+d\left(x_{2 n+1}, x_{2 n+2}\right)\right] .
\end{aligned}
$$

Thus

$$
d\left(x_{2 n+1}, x_{2 n+2}\right) \leq(I-N-P)^{-1}(M+N+P) d\left(x_{2 n}, x_{2 n+1}\right)=C d\left(x_{2 n}, x_{2 n+1}\right) .
$$

We obtain that:

$$
d\left(x_{n}, x_{n+1}\right) \leq C^{n} d\left(x_{0}, x_{1}\right), \quad \text { for each } n \in \mathbb{N} .
$$

To prove that $\left(x_{n}\right)_{n \in \mathbb{N}}$ is a Cauchy sequence, we estimate $d\left(x_{n}, x_{n+p}\right)$ using the triangle inequality:

$$
\begin{aligned}
d\left(x_{n}, x_{n+p}\right) & \leq d\left(x_{n}, x_{n+1}\right)+d\left(x_{n+1}, x_{n+2}\right)+\cdots+d\left(x_{n+p-1}, x_{n+p}\right) \\
& \leq C^{n} d\left(x_{0}, x_{1}\right)+C^{n+1} d\left(x_{0}, x_{1}\right)+\cdots+C^{n+p-1} d\left(x_{0}, x_{1}\right) \\
& \leq C^{n}(I-C)^{-1} d\left(x_{0}, x_{1}\right) .
\end{aligned}
$$

Note that $(I-C)$ is nonsingular since $C$ is convergent to zero. This implies that the sequence $\left(x_{n}\right)_{n \in \mathbb{N}}$ is a Cauchy sequence. Using the fact that $(X, d)$ is complete we get that $\left(x_{n}\right)_{n \in \mathbb{N}}$ is convergent in $X$. Thus, there exists $z \in X$ such that $d\left(x_{n}, z\right) \rightarrow 0$, as $n \rightarrow \infty$.

Now, we show that $z$ is a fixed point for $f$ by esteeming $d(f(z), z)$ we obtain:

$$
d(f(z), z)-d\left(x_{2 n+2}, z\right) \leq d\left(f(z), g\left(x_{2 n+1}\right)\right)
$$

and

$$
\begin{aligned}
d\left(f(z), g\left(x_{2 n+1}\right)\right) \leq & M d\left(z, x_{2 n+1}\right)+N\left[d(z, f(z))+d\left(x_{2 n+1}, g\left(x_{2 n+1}\right)\right)\right] \\
& +P\left[d\left(z, g\left(x_{2 n+1}\right)\right)+d\left(x_{2 n+1}, f(z)\right)\right] .
\end{aligned}
$$

We obtain:

$$
\begin{aligned}
d(f(z), z)-d\left(x_{2 n+2}, z\right) \leq & M d\left(z, x_{2 n+1}\right)+N\left[d(z, f(z))+d\left(x_{2 n+1}, x_{2 n+2}\right)\right] \\
& +P\left[d\left(z, x_{2 n+2}\right)+d\left(x_{2 n+1}, z\right)+d(z, f(z))\right] .
\end{aligned}
$$

Passing to the limit and taking into account that $(I-N-P)$ is nonsingular and $(I-$ $N-P)^{-1} \in \mathcal{M}_{n \times n}\left(\mathbb{R}_{+}\right)$we get that $z$ is a fixed point for $f$. 
Let us show that $g(z)=z$ using the condition (iii), we have:

$$
\begin{aligned}
d(z, g(z)) & =d(f(z), g(z)) \\
& \leq M d(z, z)+N[d(z, f(z))+d(z, g(z))]+P[d(z, g(z))+d(z, f(z))],
\end{aligned}
$$

we obtain:

$$
(I-N-P) d(z, g(z)) \leq 0 .
$$

Note that $(I-N-P)$ is nonsingular and $(I-N-P)^{-1} \in \mathcal{M}_{n \times n}\left(\mathbb{R}_{+}\right)$we conclude that $z=g(z)$ so we obtain that $z$ is a common fixed point for $f$ and $g$.

Now, we show that $f$ and $g$ have a unique common fixed point. For this, we assume that there exists another point $w$ fixed by $f$. Using the condition (iii), we have:

$$
\begin{aligned}
d(w, z) & =d(f(w), g(z)) \\
& \leq M d(w, z)+N[d(w, f(w))+d(z, g(z))]+P[d(w, g(z))+d(z, f(w))]
\end{aligned}
$$

We obtain:

$$
(I-M-2 P) d(w, z) \leq 0 .
$$

Taking into account that $(I-M-2 P)$ is nonsingular and $(I-M-2 P)^{-1} \in \mathcal{M}_{n \times n}\left(\mathbb{R}_{+}\right)$ this implies that $z$ is a unique common fixed point for $f$ and $g$.

If $n=1$ in the previous theorem, then we get the concept of the usual metric space, in this case we have:

3.2. Corollary. Let $(X, d)$ be a complete metric space. Assume that the operators $f, g$ : $X \rightarrow X$ satisfy the following conditions:

there exists constants $M, N, P \in \mathbb{R}_{+}$with:

(i) $d(f(x), g(y)) \leq M d(x, y)+N[d(x, f(x))+d(y, g(y))]+P[d(x, g(y))+d(y, f(x))]$, for all $x, y \in X$;

(ii) $0<C<1$ with $C=\frac{M+N+P}{1-N-P}$.

Then:

(1) $f$ and $g$ have a common fixed point $z$ in $X$.

(2) If, in addition, $M+2 P<1$ then $z$ is unique.

As an application of the previous results we present an existence theorem for a system of operatorial equations.

3.3. Theorem. Let $(X,||$.$) be a Banach space and let f, g: X \times X \rightarrow X$ be two operators. Suppose that there exist $m_{i j}, n_{i j}, p_{i j} \in \mathbb{R}_{+}$, with:

$$
M=\left(\begin{array}{ll}
m_{11} & m_{12} \\
m_{21} & m_{22}
\end{array}\right), \quad N=\left(\begin{array}{ll}
n_{11} & n_{12} \\
n_{21} & n_{22}
\end{array}\right), \quad P=\left(\begin{array}{ll}
p_{11} & p_{12} \\
p_{21} & p_{22}
\end{array}\right)
$$

such that, for each $x=\left(x_{1}, x_{2}\right), y=\left(y_{1}, y_{2}\right) \in X \times X$, one has:

(1) $\left|f_{1}\left(x_{1}, x_{2}\right)-g_{1}\left(y_{1}, y_{2}\right)\right| \leq m_{11}\left|x_{1}-y_{1}\right|+m_{12}\left|x_{2}-y_{2}\right|+n_{11}\left(\left|x_{1}-f_{1}\left(x_{1}, x_{2}\right)\right|+\right.$ $\left.\left|y_{1}-g_{1}\left(y_{1}, y_{2}\right)\right|\right)+n_{12}\left(\left|x_{2}-f_{2}\left(x_{1}, x_{2}\right)\right|+\left|y_{2}-g_{2}\left(y_{1}, y_{2}\right)\right|\right)+p_{11}\left(\left|x_{1}-g_{1}\left(y_{1}, y_{2}\right)\right|+\mid y_{1}-\right.$ $\left.f_{1}\left(x_{1}, x_{2}\right) \mid\right)+p_{12}\left(\left|x_{2}-g_{2}\left(y_{1}, y_{2}\right)\right|+\left|y_{2}-f_{2}\left(x_{1}, x_{2}\right)\right|\right)$

(2) $\left|f_{2}\left(x_{1}, x_{2}\right)-g_{2}\left(y_{1}, y_{2}\right)\right| \leq m_{21}\left|x_{1}-y_{1}\right|+m_{22}\left|x_{2}-y_{2}\right|+n_{21}\left(\left|x_{1}-f_{1}\left(x_{1}, x_{2}\right)\right|+\right.$ $\left.\left|y_{1}-g_{1}\left(y_{1}, y_{2}\right)\right|\right)+n_{22}\left(\left|x_{2}-f_{2}\left(x_{1}, x_{2}\right)\right|+\left|y_{2}-g_{2}\left(y_{1}, y_{2}\right)\right|\right)+p_{21}\left(\left|x_{1}-g_{1}\left(y_{1}, y_{2}\right)\right|+\mid y_{1}-\right.$ $\left.f_{1}\left(x_{1}, x_{2}\right) \mid\right)+p_{22}\left(\left|x_{2}-g_{2}\left(y_{1}, y_{2}\right)\right|+\left|y_{2}-f_{2}\left(x_{1}, x_{2}\right)\right|\right)$.

In addition, we assume that the matrix $(I-N-P)$ is nonsingular and $(I-N-$ 
$P)^{-1} \in \mathcal{M}_{n \times n}\left(\mathbb{R}_{+}\right)$and $C$ is convergent to zero.

Then, the system

$$
\begin{aligned}
& u_{1}=f_{1}\left(u_{1}, u_{2}\right)=g_{1}\left(u_{1}, u_{2}\right), \\
& u_{2}=f_{2}\left(u_{1}, u_{2}\right)=g_{2}\left(u_{1}, u_{2}\right),
\end{aligned}
$$

has at least one solution $z \in X \times X$. Moreover, if, in addition, the matrix $(I-M-2 P)$ is nonsingular and $(I-M-2 P)^{-1} \in \mathcal{M}_{n \times n}\left(\mathbb{R}_{+}\right)$, then the above solution is unique.

Proof. Consider $E=X \times X$ and the operators $f, g: E \rightarrow E$ given by the expression:

$$
\begin{aligned}
& f\left(x_{1}, x_{2}\right)=\left(f_{1}\left(x_{1}, x_{2}\right), f_{2}\left(x_{1}, x_{2}\right)\right) \\
& g\left(y_{1}, y_{2}\right)=\left(g_{1}\left(y_{1}, y_{2}\right), g_{2}\left(y_{1}, y_{2}\right)\right)
\end{aligned}
$$

Then our system is now represented as a fixed point equation of the following form: $w=f(w)=g(w), w \in E$. Notice also that the conditions $(1)+(2)$ can be jointly represented as follows:

$$
\begin{aligned}
\|f(x)-g(y)\| \leq & M\|x-y\|+N(\|x-f(x)\|+\|y-g(y)\|) \\
& +P(\|x-g(y)\|+\|y-f(x)\|) \text { for all } x, y \in X \times X .
\end{aligned}
$$

Hence, theorem (3.1) applies in $(E, d)$, with $d(u, v)=\|u-v\|=\left(\begin{array}{l}\left|u_{1}-v_{1}\right| \\ \left|u_{2}-v_{2}\right|\end{array}\right)$.

Now, we present another result in the case of a generalized metric space but endowed with two metrics.

3.4. Theorem. Let $(X, \delta)$ be a complete generalized metric space and d another vectorvalued metric on $X$. Assume that the operators $f, g: X \rightarrow X$ satisfy the following conditions:

(a): There exists a matrix $U \in \mathcal{M}_{n \times n}\left(\mathbb{R}_{+}\right)$such that $\delta(x, y) \leq U \cdot d(x, y)$, for all $x, y \in X$;

(b): $f$ is $(\delta, \delta)$-continuous;

(c): There exists matrices $M, N, P \in \mathcal{M}_{n \times n}\left(\mathbb{R}_{+}\right)$with:

(i): $(I-N-P)$ is nonsingular and $(I-N-P)^{-1} \in \mathcal{M}_{n \times n}\left(\mathbb{R}_{+}\right)$;

(ii): $C$ is convergent toward zero, where $C=(I-N-P)^{-1}(M+N+P)$

(iii): $d(f(x), g(y)) \leq M d(x, y)+N[d(x, f(x))+d(y, g(y))]+P[d(x, g(y))+$ $d(y, f(x))]$, for all $x, y \in X$.

Then:

(1) For any $x_{0} \in X$ we have $\delta\left(f^{k}\left(x_{0}\right), z\right) \rightarrow 0$, as $k \rightarrow \infty$, where $z$ is a fixed point for $f$ and $g$.

(2) If, in addition, $(I-M-2 P)$ is nonsingular and $(I-M-2 P)^{-1} \in \mathcal{M}_{n \times n}\left(\mathbb{R}_{+}\right)$, then $z$ is a unique common fixed point of $f$ and $g$.

Proof. Let $x_{0}$ be some point in $X$, we consider $\left(x_{n}\right)_{n \in \mathbb{N}}$ the sequence of successive approximations for $f$ and $g$, defined by:

$$
\begin{array}{ll}
x_{2 n+1}=f\left(x_{2 n}\right), & n=0,1, \ldots \\
x_{2 n+2}=g\left(x_{2 n+1}\right), & n=0,1, \ldots
\end{array}
$$


We have:

$$
\begin{aligned}
d\left(x_{2 n}, x_{2 n+1}\right)= & d\left(g\left(x_{2 n-1}\right), f\left(x_{2 n}\right)\right) \\
\leq & M d\left(x_{2 n-1}, x_{2 n}\right)+N\left[d\left(x_{2 n}, f\left(x_{2 n}\right)\right)+d\left(x_{2 n-1}, g\left(x_{2 n-1}\right)\right)\right] \\
& +P\left[d\left(x_{2 n}, g\left(x_{2 n-1}\right)\right)+d\left(x_{2 n-1}, f\left(x_{2 n}\right)\right)\right] \\
= & M d\left(x_{2 n-1}, x_{2 n}\right)+N\left[d\left(x_{2 n}, x_{2 n+1}\right)+d\left(x_{2 n-1}, x_{2 n}\right)\right]+P d\left(x_{2 n-1}, x_{2 n+1}\right) \\
\leq & M d\left(x_{2 n-1}, x_{2 n}\right)+N\left[d\left(x_{2 n}, x_{2 n+1}\right)+d\left(x_{2 n-1}, x_{2 n}\right)\right] \\
& +P\left[d\left(x_{2 n-1}, x_{2 n}\right)+d\left(x_{2 n}, x_{2 n+1}\right)\right] .
\end{aligned}
$$

This implies that:

$$
d\left(x_{2 n}, x_{2 n+1}\right) \leq(I-N-P)^{-1}(M+N+P) d\left(x_{2 n-1}, x_{2 n}\right)=C d\left(x_{2 n-1}, x_{2 n}\right) .
$$

Similarly, we have:

$$
\begin{aligned}
d\left(x_{2 n+1}, x_{2 n+2}\right)= & d\left(f\left(x_{2 n}\right), g\left(x_{2 n+1}\right)\right) \\
\leq & M d\left(x_{2 n}, x_{2 n+1}\right)+N\left[d\left(x_{2 n}, f\left(x_{2 n}\right)\right)+d\left(x_{2 n+1}, g\left(x_{2 n+1}\right)\right)\right] \\
& +P\left[d\left(x_{2 n}, g\left(x_{2 n+1}\right)\right)+d\left(x_{2 n+1}, f\left(x_{2 n}\right)\right)\right] \\
= & M d\left(x_{2 n}, x_{2 n+1}\right)+N\left[d\left(x_{2 n}, x_{2 n+1}\right)+d\left(x_{2 n+1}, x_{2 n+2}\right)\right]+P d\left(x_{2 n}, x_{2 n+2}\right) \\
\leq & M d\left(x_{2 n}, x_{2 n+1}\right)+N\left[d\left(x_{2 n}, x_{2 n+1}\right)+d\left(x_{2 n+1}, x_{2 n+2}\right)\right] \\
& +P\left[d\left(x_{2 n}, x_{2 n+1}\right)+d\left(x_{2 n+1}, x_{2 n+2}\right)\right] .
\end{aligned}
$$

Thus

$$
d\left(x_{2 n+1}, x_{2 n+2}\right) \leq(I-N-P)^{-1}(M+N+P) d\left(x_{2 n}, x_{2 n+1}\right)=C d\left(x_{2 n}, x_{2 n+1}\right) .
$$

We obtain that:

$$
d\left(x_{n}, x_{n+1}\right) \leq C^{n} d\left(x_{0}, x_{1}\right), \quad \text { for each } n \in \mathbb{N} .
$$

To prove that $\left(x_{n}\right)_{n \in \mathbb{N}}$ is a Cauchy sequence, we estimate $d\left(x_{n}, x_{n+p}\right)$ using the triangle inequality:

$$
\begin{aligned}
d\left(x_{n}, x_{n+p}\right) & \leq d\left(x_{n}, x_{n+1}\right)+d\left(x_{n+1}, x_{n+2}\right)+\cdots+d\left(x_{n+p-1}, x_{n+p}\right) \\
& \leq C^{n} d\left(x_{0}, x_{1}\right)+C^{n+1} d\left(x_{0}, x_{1}\right)+\cdots+C^{n+p-1} d\left(x_{0}, x_{1}\right) \\
& =C^{n}\left[I+C+\cdots+C^{p-1}\right] d\left(x_{0}, x_{1}\right) \\
& \leq C^{n}(I-C)^{-1} d\left(x_{0}, x_{1}\right) .
\end{aligned}
$$

Note that $(I-C)$ is nonsingular since $C$ is convergent to zero. This implies that the sequence $\left(x_{n}\right)_{n \in \mathbb{N}}$ is $d$-Cauchy. It follows from (a) that $\left(x_{n}\right)_{n \in \mathbb{N}}$ is $\delta$-Cauchy sequence. Since $(X, \delta)$ is a complete generalized metric space, there exists $z \in X$ such that $\delta\left(x_{2 n+1}, z\right) \rightarrow 0$, as $n \rightarrow \infty$.

By (b), we have $\delta\left(f\left(x_{2 n}\right), f(z)\right)=\delta\left(x_{2 n+1}, f(z)\right)$. Hence we have $z=f(z)$. Thus $z$ is a fixed point for $f$ and

$$
\delta\left(f^{n}\left(x_{0}\right), z\right) \rightarrow 0, \text { as } n \rightarrow \infty .
$$

The rest of the proof follows as in theorem (3.1).

\section{References}

[1] G. E. Hardy and T. D. Rogers, A generalization of a fixed point theorem of Reich, Canad. Math. Bull. 16, 201-206, 1973.

[2] R. Kannan, Some remarks on fixed points, Bull. Calcutta Math. Soc. 60, 71-76, 1960.

[3] D. O'Regan, R. Precup, Continuation theory for contractions on spaces with two vectorvalued metrics, Applicable Analysis. 82, 131-144, 2003.

[4] A. I. Perov, On the Cauchy problem for a system of ordinary differential equations, Pviblizhen. Met. Reshen. Differ. Uvavn. 2, 115-134, 1964. 
[5] R. Precup, The role of matrices that are convergent to zero in the study of semilinear operator systems, Mathematical and Computer Modelling, 49, no. 3-4, 703-708, 2009.

[6] S. Reich, Some results concerning contraction mappings, Canad. Math. Bull. 14, 121-124, 1971.

[7] I. A. Rus, Principles and applications of the fixed Point Theory, Dacia, Cluj-Napoca, Romania, 1979.

[8] M. Turinici, Finite-dimensional vector contractions and their fixed points, Studia Universitatis Babeş-Bolyai. Mathematica, 35, no. 1, 30-42, 1990.

[9] R. S. Varga, Matrix Iterative Analysis, vol. 27 of Springer Series in Computational Mathematics Springer, Berlin, Germany, 2000. 